\title{
The management of breast implant-associated anaplastic large cell lymphoma in the setting of pregnancy: seeking for clinical practice guidelines
}

\author{
Rossella Elia ${ }^{1}$, Michele Maruccia ${ }^{1}$, Aurelia De Pascale ${ }^{1}$, Arianna Di Napoli ${ }^{2}$, Giuseppe Ingravallo ${ }^{3}$, \\ Giuseppe Giudice ${ }^{1}$ \\ ${ }^{1}$ Division of Plastic and Reconstructive Surgery, Department of Emergency and Organ Transplantation, University of Bari Aldo Moro, Bari; \\ ${ }^{2}$ Pathology Unit, Department of Clinical and Molecular Medicine, Sant'Andrea Hospital, Sapienza University of Rome, Roma; ${ }^{3}$ Section of \\ Pathology, Department of Emergency and Organ Transplantation, University of Bari Aldo Moro, Bari, Italy
}

Breast implant-associated anaplastic large cell lymphoma (BIA-ALCL) is a recently described form of T-cell non-Hodgkin lymphoma now formally recognized by the World Health Organization classification of lymphoid neoplasms. The aim of this paper is to report the first case of BIA-ALCL diagnosed in a pregnant patient. It is well known that BIA-ALCL appears as an indolent lymphoma with a good prognosis when diag-nosed at early stages and clinical guidelines for its management have been clearly published. Nevertheless, they lack a standardized approach for BIA-ALCL during pregnancy. With limited experience in our case, treatment has been safely postponed after term without affecting patient's overall prognosis and without fetal complication. The fact that the disease was diagnosed at an early stage (stage I) undoubtedly influenced the course of treatment. A multidisciplinary approach weighing the risks and benefits of treatment is of paramount importance in order to ensure the best possible outcome for both the mother and her child and clinical update guidelines should be issued.

Keywords Lymphoma, large-cell, anaplastic / Lymphoma / Pregnancy / Breast implants / World Health Organization
Correspondence: Rossella Elia Division of Plastic and Reconstructive Surgery, Department of Emergency and Organ Transplantation, University of Bari Aldo Moro, 11 Piazza Giulio Cesare, Bari 70124, Italy Tel: $+39-3409616476$ Fax: $+39-805714030$

E-mail: rossellaelia4@gmail.com

\section{INTRODUCTION}

Breast implant-associated anaplastic large cell lymphoma (BIAALCL) is a rare form of ALCL, described first in 1997 and formally recognized as a specific entity by World Health Organization in 2016 [1]. Since the first case, a constant increase in BIAALCL diagnosis has been recorded, reflecting a better recognition of the disease by surgeons and pathologists $[2,3]$.

The pathogenesis of BIA-ALCL remains elusive. It is postulat- ed that local chronic inflammation elicitated by implant products or bacterial infection may promote the activation and proliferation of $\mathrm{T}$ cells which, by the acquisition of molecular alterations transform into lymphoma cells [3-5].

National Comprehensive Cancer Network consensus guidelines have been established and widely adopted for the diagnosis and management of BIA-ALCL [6].

According to the TNM staging system, patients with stage I disease have lymphoma evidenced into the capsule of the im- 
plant. Those with stage IIA disease have disease limited to the breast but with evidence of lymphoma invading beyond the capsule. Patients with stage IIB disease have involvement of a single regional lymph node. Any disease outside of the ipsilateral breast and regional lymph node basins is considered stage IV disease. Surgical ablation with explantation and capsulectomy is frequently curative with disease confined to the capsule.

The aim of this paper is to report the first case of BIA-ALCL

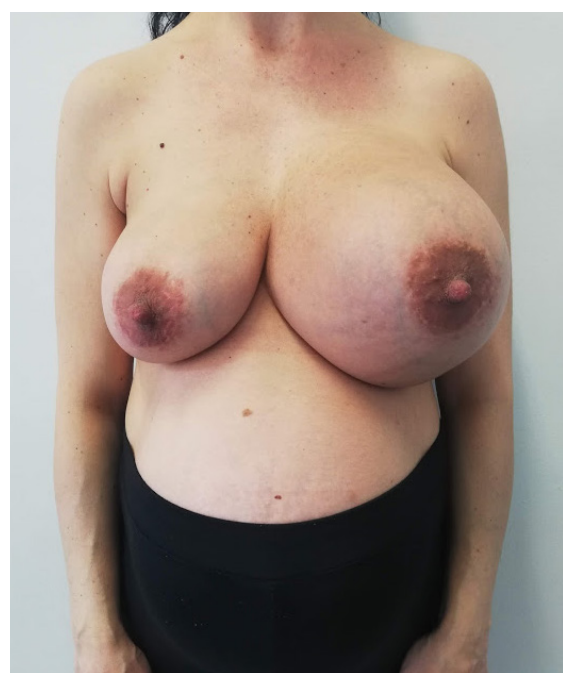

Fig. 1. Late periprosthetic breast seroma in a 40 -year-old 25 weeks pregnant patient, suggesting for breast implant-associated anaplastic large cell lymphoma. diagnosed in a pregnant patient, raising questions on how to manage this disease in the setting of pregnancy.

\section{CASE}

A 40-year-old 25 weeks pregnant (patient) was admitted to our clinic presenting a large spontaneous periprosthetic fluid collection at the left breast, occurring 12 years following bilateral cosmetic sub-glandular implantation with a textured surface silicone gel-filled breast implant (Fig. 1). At the time of presentation, no palpable mass nor lymphadenopathy was observed.

She referred that clinical and instrumental (ultrasonography and magnetic resonance imaging) diagnosis of a left breast seroma dated back to 2 years before and had been treated conservatively through ago aspiration and anti-inflammatory drugs. Cultural test performed through fine needle aspiration 6 months before was negative for any bacterial/fungal infection and cytology test was negative for malignancy.

Fine needle aspiration was repeated during our first consultation and $50 \mathrm{~mL}$ of periprosthetic fluid sample was sent for cytological examination. Cell smear and cell blocks showed an infiltrate composed predominantly of large to medium-sized atypical cells with occasional mitotic figures and apoptotic debris in a fibrinous/necrotic background. By immunocytochemistry, the atypical cells were positive for CD30, CD4, IRF4, and negative for CD3, CD8, CD20, CD68, ALK (anaplastic lymphoma ki-
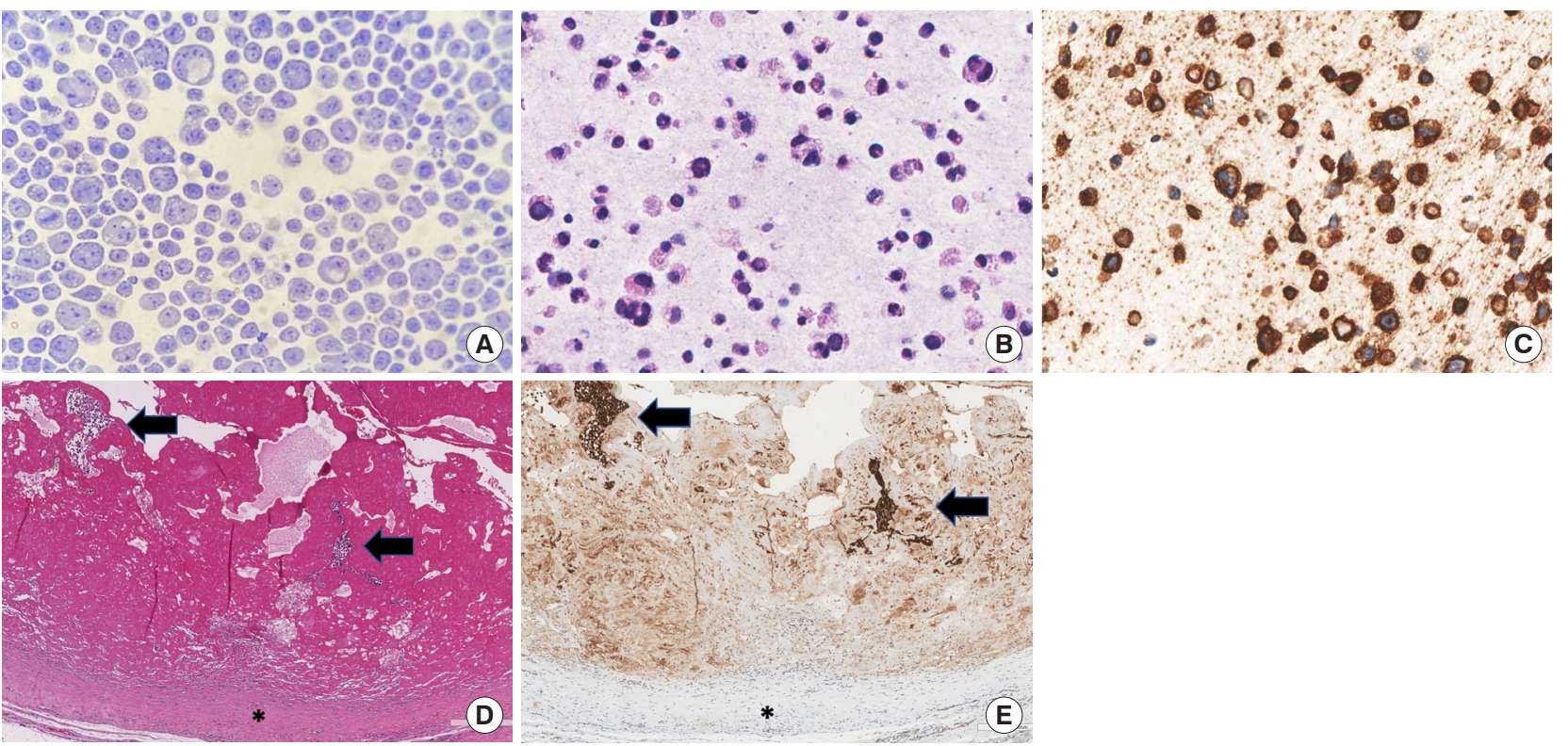

Fig. 2. Cytological and histological images of peri-implant left breast seroma $(A-C)$ and homolateral fibrous capsule (D, E). Papanicolaou stained cell smear $(A, \times 400)$ and hematoxylin and eosin-stained section of cell block $(B, \times 400)$ showed large pleomorphic cells with irregularly shaped nuclei and binucleated elements immunostained for $\operatorname{CD} 30\left(C_{1} \times 400\right)$. Histological examinations of the capsule revealed clusters of large atypical cells within a fibrinous exudate (black arrows) on the inner surface of the capsule (black asterisk) $(D, H \& E, \times 100)$. The anaplastic cells were highlighted by CD30 stain $\left(E_{1} \times 100\right)$. 


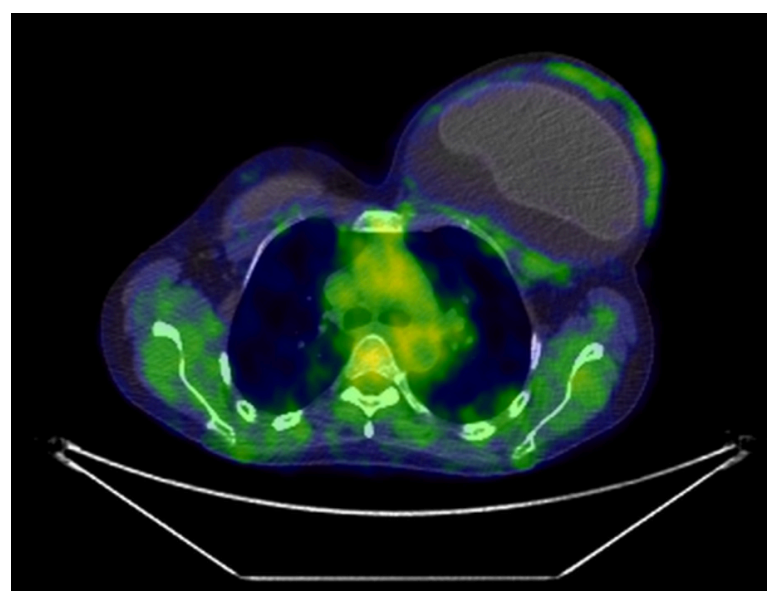

Fig. 3. An effusion-limited (stage IA) left breast implant-associated anaplastic large cell lymphoma is shown on an axial ${ }^{18} \mathrm{~F}$-fluorodeoxyglucose positron emission tomography/computed tomographic image with the increased metabolic activity of the left capsule.

nase) and EMA (epithelial membrane antigen) consistent with a diagnosis of BIA-ALCL (Fig. 2).

Once the diagnosis of BIA-ALCL was established, a multidisciplinary team including oncologist, pathologist, hematologist and gynecologist recommended a positron emission tomography computed tomography (PET/CT) scan for staging and surgical intervention when the patient would have reached full term pregnancy. Meanwhile, a breast and axillary ultrasonography was performed, which did not evidence focal alteration outside the periprosthetic capsule.

Twenty days after childbirth, PET/CT demonstrated an augmented glucose metabolism at the left breast with no associated capsular mass nor chest, lymph node or visceral involvement (stage IA according to the MD Anderson TNM classification) (Fig. 3) [7].

Surgical treatment with en bloc explantation of the implant and the surrounding fibrous capsule was performed bilaterally. Surgical specimens were oriented and inked for pathological processing as recently proposed [8]. Postoperative course was uneventful.

Histological examination showed the presence of $\mathrm{CD} 30+$, CD4+, CD3-, ALK- anaplastic large lymphoma cells admixed with abundant granular and necrotic material on the luminal surface of the left capsule confirming stage I disease. Lymphoma cells were not detected on the right capsule.

Patient is actually followed up according to National Comprehensive Cancer Network guidelines (history and physical every 3 to 6 months for 2 years and PET scan every 6 months for 2 years and then only if clinically indicated) [6] in an outpatient setting and no tumor progression has been observed in 12 months of follow-up.

\section{DISCUSSION}

Approximately one of every 1,000 deliveries is complicated by cancer. Lymphoma ranks fourth after cervical and breast cancers and melanoma, with an approximate incidence of one per 6,000 [9]. To our knowledge, only seven cases of ALCL have been reported in the setting of pregnancy [9-13] whereas no case of BIA-ALCL has been described so far.

It is well known that BIA-ALCL appears as an indolent lymphoma with a good prognosis when diagnosed at early stages and clinical guidelines for its management have been clearly published [6]. Nevertheless, they lack a standardized approach for BIA-ALCL as well as for ALK-positive systemic ALCL during pregnancy [14].

In our case, the decision to postpone the treatment after childbirth did not change the patient's stadiation and prognosis. Indeed PET/CT scan, which was not performed before childbirth as PET with ${ }^{18} \mathrm{~F}$-fluorodeoxyglucose tracer exposes the fetus to a high dose of radiation and is not recommended during pregnancy [15], confirmed a stage IA disease. Patient was treated with complete surgical bilateral excision of implants and their surrounding fibrous capsule because an estimated $4.6 \%$ of BIAALCL cases to date have demonstrated incidental ALCL in the contralateral breast implant [3]. Conversely, there is no clear role for radical mastectomy or sentinel lymph node biopsy [7]. Full axillary dissection has been used rarely for gross involvement of multiple lymph nodes.

After the diagnosis and surgical treatment of BIA-ALCL, it has been recommended that only autologous reconstruction would be offered until further evidence is gathered [16-19]. However, our patient refused any autologous breast augmentation.

Our case adds to the limited available evidence on this topic, highlighting how BIA-ALCL should be considered for diagnosis in the presence of spontaneous late-onset periprosthetic seroma even during pregnancy and the histological diagnostic features of the disease do not differ in a pregnant woman.

Moreover, this paper further supports the idea that delivery at term is ideal in the management of pregnant patients with malignancy having demonstrated no fetal complications. Treatment has been safely postponed after term without affecting patient's overall prognosis, endorsing the evidence that pregnancy itself has not been shown to worsen a malignancy [20].

Clinical and molecular evidences are warranted to understand whether a more advanced stage of the disease or an earlier diagnosis during pregnancy would have been changed the therapeutic algorithm.

With limited experience in the treatment of BIA-ALCL during pregnancy, a multidisciplinary approach meticulously weighing 
the risks and benefits of treatment is of paramount importance in order to ensure the best possible outcome for both the mother and her child and clinical update guidelines should be issued.

\section{NOTES}

\section{Conflict of interest}

No potential conflict of interest relevant to this article was reported.

\section{Ethical approval}

The study was performed in accordance with the principles of the Declaration of Helsinki. Written informed consent was obtained.

\section{Patient consent}

The patient provided written informed consent for the publication and the use of her images.

\section{Author contribution}

Conceptualization: R Elia. Data curation: M Maruccia, A Di Napoli, G Ingravallo. Methodology: R Elia. Writing - original draft: R Elia, A De Pascale. Writing - review \& editing: R Elia, M Maruccia, G Ingravallo.

\section{ORCID}

Rossella Elia https://orcid.org/0000-0002-6094-5159

Michele Maruccia https://orcid.org/0000-0002-2122-2732 Arianna Di Napoli https://orcid.org/0000-0002-3159-5380 Giuseppe Ingravallo https://orcid.org/0000-0002-4792-3545

\section{REFERENCES}

1. Swerdlow SH, Campo E, Pileri SA, et al. The 2016 revision of the World Health Organization classification of lymphoid neoplasms. Blood 2016;127:2375-90.

2. Campanale A, Spagnoli A, Lispi L, et al. The crucial role of surgical treatment in BIA-ALCL prognosis in early- and advanced-stage patients. Plast Reconstr Surg 2020;146:530e538e.

3. Clemens MW, Brody GS, Mahabir RC, et al. How to diagnose and treat breast implant-associated anaplastic large cell lymphoma. Plast Reconstr Surg 2018; 141:586e-599e.

4. Di Napoli A, Jain P, Duranti E, et al. Targeted next generation sequencing of breast implant-associated anaplastic large cell lymphoma reveals mutations in JAK/STAT signalling pathway genes, TP53 and DNMT3A. Br J Haematol 2018; 180:741-4.
5. Di Napoli A, De Cecco L, Piccaluga PP, et al. Transcriptional analysis distinguishes breast implant-associated anaplastic large cell lymphoma from other peripheral T-cell lymphomas. Mod Pathol 2019;32:216-30.

6. Clemens MW, Jacobsen ED, Horwitz SM. 2019 NCCN consensus guidelines on the diagnosis and treatment of breast implant-associated anaplastic large cell lymphoma (BIA-ALCL). Aesthet Surg J 2019;39(Suppl_1):S3-13.

7. Clemens MW, Medeiros LJ, Butler CE, et al. Complete surgical excision is essential for the management of patients with breast implant-associated anaplastic large-cell lymphoma. J Clin Oncol 2016;34:160-8.

8. Lyapichev KA, Pina-Oviedo S, Medeiros LJ, et al. A proposal for pathologic processing of breast implant capsules in patients with suspected breast implant anaplastic large cell lymphoma. Mod Pathol 2020;33:367-79.

9. Lesesve JF, Buisine J, Gregoire MJ, et al. Leukaemic small cell variant anaplastic large cell lym-phoma during pregnancy. Clin Lab Haematol 2000;22:297-301.

10. Kato M, Ichimura K, Hayami Y, et al. Pregnancy-associated cytotoxic lymphoma: a report of 4 cases. Int J Hematol 2001; 74:186-92.

11. Iyengar P, Reid-Nicholson M, Moreira AL. Pregnancy-associated anaplastic large-cell lymphoma of the breast: a rare mimic of ductal carcinoma. Diagn Cytopathol 2006;34:298302.

12. Alalade AO, Odejinmi FO. A rare and potentially fatal cause of pelvic pain in pregnancy: anaplastic large cell lymphoma. J Obstet Gynaecol 2006;26:691-2.

13. Kisacik B, Akdogan A, Maras Y, et al. Anaplastic large cell lymphoma presenting with symmetric polyarthritis in pregnancy. Rheumatol Int 2008;28:909-11.

14. Peccatori FA, Azim HA Jr, Pruneri G, et al. Management of anaplastic large-cell lymphoma during pregnancy. J Clin Oncol 2009;27:e75-7.

15. Orecchia R, Lucignani G, Tosi G. Prenatal irradiation and pregnancy: the effects of diagnostic imaging and radiation therapy. Recent Results Cancer Res 2008;178:3-20.

16. Santanelli di Pompeo F, Laporta R, Sorotos M, et al. Breast implant-associated anaplastic large cell lymphoma: proposal for a monitoring protocol. Plast Reconstr Surg 2015;136: 144e-151e.

17. Maruccia M, Elia R, Gurrado A, et al. Skin-reducing mastectomy and pre-pectoral breast reconstruction in large ptotic breasts. Aesthetic Plast Surg 2020;44:664-72.

18. Giudice G, Maruccia M, Vestita M, et al. The medial-central septum based mammaplasty: a reliable technique to preserve nippleareola complex sensitivity in post bariatric pa- 
tients. Breast J 2019;25:590-6.

19. Bucaria V, Elia R, Maruccia M, et al. Methylene blue: a color test for a quality de-epithelialization. Aesthetic Plast Surg 2018;42:1434-5.
20. Zagouri F, Dimitrakakis C, Marinopoulos S, et al. Cancer in pregnancy: disentangling treatment modalities. ESMO Open 2016;1:e000016. 